PROCEEDINGS OF THE

AMERICAN MATHEMATICAL SOCIETY

Volume 125, Number 2, February 1997, Pages 547-554

S 0002-9939(97)03614-9

\title{
A MOVING-KNIFE SOLUTION TO THE FOUR-PERSON ENVY-FREE CAKE-DIVISION PROBLEM
}

\author{
STEVEN J. BRAMS, ALAN D. TAYLOR, AND WILLIAM S. ZWICKER \\ (Communicated by Andreas R. Blass)
}

\begin{abstract}
We present a moving-knife procedure, requiring only 11 cuts, that produces an envy-free allocation of a cake among four players and discuss possible extensions to five players.
\end{abstract}

\section{INTRODUCTION}

We assume the reader is familiar with the general problem, dating back to Steinhaus $[\mathrm{S}]$, of dividing a cake among $n$ people so that each is "satisfied" with the piece he or she receives. General background can be found in [BT 1], [BT 2], or [BTZ]. There are several possible notions of satisfaction, but the only one that we will be concerned with here is the one wherein each player thinks he or she receives a piece of cake at least tied for largest. Such an allocation is said to be envy-free.

Around 1960, John Selfridge and John Conway independently found a discrete scheme for producing an envy-free allocation among three players (see [BT 1] or [BT 2]). Two decades later, Walter Stromquist [St] found a moving-knife procedure for producing such an allocation, but it also worked only for three or fewer players. In [BTZ] we survey several moving-knife schemes, five of which produce an envy-free allocation among three players.

The problem of finding a constructive procedure for producing an envy-free allocation among four or more players was open until 1992 (see [BT 1], [BT 2] and [BT 3]). The solution to that problem, however, involved an algorithm that was of unbounded length, even for the case $n=4$. That is, even for four players the number of cuts necessary to construct an envy-free division, via that algorithm, could be made arbitrarily large by a suitable choice of the measures corresponding to the players' preferences.

In the present paper we produce a moving-knife scheme that yields an envy-free allocation among four players. This procedure is based on a scheme due to A. K. Austin [A] that we sketch in Section 2 as part of the proof of our main result. In Section 3, we show how a modification of the scheme in Section 2 yields an envyfree procedure that requires only eleven cuts. Whether or not this is best possible is an open question. Finally, in Section 4, we show that - given an oracle (defined later) for producing a minimal extension of Austin's scheme - we can extend the envy-free scheme of Section 2 to the case of five players.

Received by the editors December 20, 1994 and, in revised form, August 29, 1995.

1991 Mathematics Subject Classification. Primary 90D10; Secondary 62C20.

(C)1997 American Mathematical Society 
In the tradition of the subject, we offer no definition of "moving-knife scheme" or of "discrete scheme"; the reader is supposed to know one when he or she sees one. However, in Section 5 we argue that formalizations - at least partial ones - will be needed to settle some issues raised in this paper.

\section{THE ENVY-FREE SCHEME FOR FOUR PLAYERS}

Theorem 1. There exists a moving-knife procedure that produces an envy-free allocation of a cake among four players.

Proof. We make use of a two-person moving-knife procedure discovered by A. K. Austin in 1982 [A]. In this procedure, each of the two players has a strategy that will guarantee him or her a piece of cake of size exactly $1 / 2$. Briefly, the scheme goes as follows.

A referee moves a single knife from the left edge of the cake toward the right edge of the cake until one of the players (assume it is player 1) calls "stop" because he or she thinks the knife is at the half-way point. Player 1 now places a second knife back at the left edge, and then simultaneously moves both knives to the right in such a way that the piece between the two knives remains of size $1 / 2$ in player 1 's estimation.

In player 2's estimation, the piece between the two knives is going from less than $1 / 2$ to greater than $1 / 2$, since it ends as the complement of what it began as. Thus, with an appropriate continuity assumption, at some point, player 2 thinks the piece between the two knives is of size exactly $1 / 2$, and he or she can call "stop" at this point.

Of course, if these same two players apply Austin's scheme to each of the two pieces arrived at above, the result is a partition of the cake into four pieces that both players agree are all of size $1 / 4$. With this, we can now give the envy-free procedure for four players.

Stage 1: Players 1 and 2 begin the procedure by using Austin's scheme to divide the cake into four pieces that they both agree are all of size $1 / 4$. Player 3 now trims at most one of the four pieces to create at least a two-way tie for largest in his estimation. The trimmings are set aside and will be allocated later.

We now achieve an envy-free allocation of all the cake, except the trimmings, by having the players choose among the four pieces (one of which may have been trimmed) in the order: player 4, player 3 , player 2, player 1 , subject to the proviso that player 3 must take the trimmed piece, if there is one and player 4 did not take it. Notice that neither player 2 nor player 1 will envy anyone, because they each receive untrimmed pieces and think all four of the original pieces are the same size.

Stage 2: For the allocation of the trimmings, we make use of the same idea as in the Selfridge-Conway discrete scheme for $n=3$, but once again invoke Austin's scheme. The key is that player 1 (and player 2, although we will not need this fact) have an "irrevocable advantage" over whichever player took the trimmed piece in Stage 1. That is, player 1 will not envy the player with the trimmed piece, regardless of how the trimmings get allocated.

Of players 3 and 4 , let the one who took the trimmed piece be renamed as "the noncutter" and the other as "the cutter". (These names will make sense in a moment.) Thus, player 1 has an irrevocable advantage over the noncutter.

For the allocation of the trimmings, we have the cutter and player 2 invoke Austin's scheme to divide the trimmings into four pieces they agree are all the 
same size. The players then choose among these four pieces, with the noncutter choosing first, player 1 second, and then the cutter and player 2 in either order.

The resulting allocation of the whole cake is envy-free. The noncutter envies no one because he is choosing first in the allocation of the trimmings. Player 1 envies neither the cutter nor player 2 because he is choosing ahead of them. Moreover, player 1 does not envy the noncutter, even though the noncutter is choosing ahead of him, because player 1 had an irrevocable advantage over the noncutter. Finally, neither the cutter nor player 2 will envy anyone, because all four pieces into which they divided the trimmings are, in their estimation, the same size.

This completes the description of the procedure and the proof of Theorem 1.

\section{Refining the EnVy-Free scheme: Eleven Cuts}

To modify the scheme from the preceding section so that only eleven cuts are needed, we need the following lemma.

Lemma. Given two players, there is a five-cut moving-knife procedure that will divide a cake into four pieces so that:

(1) one of the players thinks it is an equal division, and

(2) the other player thinks there is at least a three-way tie for largest among the four pieces.

Proof. The procedure begins with a knife moving from the left edge of the cake toward the right edge, as in Austin's scheme. When either of the players thinks the resulting piece is of size $1 / 4$, he or she calls "stop", and the cut is made. At this point, the other player thinks that piece is of size less than, or perhaps equal to, $1 / 4$. Call this piece $A$, and assume it was player 1 who called stop.

On the remainder of the cake, player 1 now makes two marks parallel to the knife cut so as to equalize the size, in his or her estimation, of the three pieces that would result if the cuts were actually made. If player 2 thinks any one of the pieces is exactly $1 / 3$ of the remainder of the cake, we make the one or two cuts, and set this piece aside. If not, player 2 identifies two adjacent pieces, one of which he or she thinks is less than $1 / 3$ of the remainder of the cake and the other of which he or she thinks is more than $1 / 3$ of the remainder of the cake.

Two knives are now moved over these adjacent pieces by player 1, exactly as in Austin's scheme, and two cuts are made at the instant when player 2 thinks the piece between the knives is exactly $1 / 3$ of the remainder of the cake. This piece is also removed (call it $B$ ), and the remains pushed together. The two players now use Austin's scheme to divide the remains 50-50 in both their estimations. This takes at most two more cuts, and yields pieces $C$ and $D$.

Player 1 , of course, thinks that $A, B, C$, and $D$ are all the same size. Player 2 either agrees, or he or she thinks that $A$ is of size less than $1 / 4$, and that $B, C$, and $D$ are tied for largest at something greater than $1 / 4$. Notice also that pieces $A$ and $B$ are intact, whereas $C$ and $D$ have two components each, or $C$ is intact and $D$ has three components.

This completes the proof of the lemma.

With the lemma at hand, it will now be an easy matter to sketch a proof of the following.

Theorem 2. There exists a moving-knife procedure, involving at most eleven cuts, that produces an envy-free allocation of a cake among four players. 
Proof. We modify the procedure in Section 2 as follows. Players 1 and 2 use the lemma in place of the two applications of Austin's scheme. Player 3 now trims, and they choose in the order player 4 , player 3 , player 2 , and player 1 , assuming that player 2 had the three pieces (from the lemma) tied for largest and player $1 \mathrm{had}$ all four the same size. Notice that we can no longer say "players 2 and 1 choose in either order". Notice also that both player 1 and player 2 still have an irrevocable advantage over whoever got the trimmed piece.

This stage took six cuts: the five from the lemma, and one more (which we picture as a cut parallel to the other cuts) for the trimming.

For the allocation of the trimmings we have the cutter and player 2 apply the lemma to the trimmings, using at most five more cuts. The players then choose in the order: noncutter, player 1, the player who had three pieces tied for largest in this latest application of the lemma, and the remaining player (the cutter or player 2) last. Because the order of these last two was irrelevant to the argument in Section 2, we have the desired allocation.

This completes the proof of Theorem 2 .

\section{THE $n=5$ CASE With AN ORACLE}

Austin's basic moving-knife procedure, described in Section 2, yields a division of the cake into two pieces such that each of two players thinks it is a $50-50$ split. This can be generalized (see [BTZ]) to yield a moving-knife scheme that will partition a cake into $k$ pieces that each of two players thinks is an even division. No extension of Austin's scheme (or this generalization) to three players is known. However, it is shown in [BTZ] that if one had a moving-knife scheme for dividing a cake into $n$ pieces so that each of $n$ players thought it was an even division, then one could obtain a moving-knife scheme for producing an envy-free allocation among $n+1$ players.

Recursion theory considers questions of the following kind: "If it were possible for a computer to perform a computation settling question $A$, would question $B$ also have a computational solution?" If so, the result is expressed by saying that " $B$ can be answered, given an oracle for $A$." Following this recursion-theoretic terminology, we will refer to a scheme whose existence is hypothesized but not known to exist as an oracle. More specifically:

Definition. An $(n, k)$-Austin oracle is a scheme (whose existence is hypothesized but not known) that produces, for $n$ players, a division of a cake into $k$ pieces so that each player thinks it is an even division.

For any $n$ atomless probability measures on a set, and any integer $k$, Neyman's theorem $[\mathrm{N}]$ implies that there exists a partition of the set into $k$ pieces that is even according to each measure. However, the proof does not help the players actually produce such a division. Thus, an $(n, k)$-Austin oracle is a black box for "constructing" objects that are known to exist.

Note that, for example, $(2,2)$-Austin oracles are not needed if one is seeking a moving-knife procedure, since Austin's original moving-knife procedure produces a division of the kind the oracle would provide. On the other hand, the use of moving knives in the scheme in Section 2 was limited to their application to even division for two players, so this result could also be described as a discrete 4-person scheme with a $(2,2)$-Austin oracle. In fact, the argument in Section 3 extends easily to show 
that a $(2, n)$-Austin oracle is not needed for any $n$ in the context of moving-knife procedures.

With this terminology, the result from [BTZ] referred to earlier can be stated as follows:

Theorem 3. Given an $(n, n)$-Austin oracle, there is a moving-knife procedure that produces an envy-free allocation of a cake among any $n+1$ players.

A $(3,2)$-Austin oracle, wherein each of three players thinks a division is 50-50, seems to be the simplest kind not known to be superfluous in the moving-knife context. In particular, if there is a $(4,4)$-Austin oracle, then there is certainly a $(3,2)$-Austin oracle. Thus, the following theorem improves the result above in the $n=5$ case, and does so by a proof that is, in our opinion, significantly more interesting.

Theorem 4. Given a $(3,2)$-Austin oracle, there exists a moving-knife procedure that produces an envy-free allocation of a cake among five players.

The proof of the theorem requires two lemmas.

Lemma I. Assume we have a $(3,2)$-Austin oracle, a piece $C$ of cake, and five players (numbered 1 through 5). Then there exists a moving-knife procedure that produces two disjoint pieces of cake, $T_{1}$ and $T_{2}$ ("trimmings"), and an envy-free allocation of $C-\left(T_{1} \cup T_{2}\right)$ among the five players, so that for some $i, j$ with $i, j \neq 2$ (although $i=j$ is possible), player 1 thinks his piece is at least as large as player $i$ 's piece with $T_{1}$ added to it, and at least as large as player $j$ 's piece with $T_{2}$ added to it.

Proof. Players 1 and 2 begin the procedure by using the generalized version of Austin's scheme, alluded to at the beginning of Section 4, to divide the cake into five pieces that they agree are all the same size. (In particular, this requires no oracle.) Player 3 now lines up the five pieces - from largest to smallest in his estimation - as $A, B, C, D$, and $E$.

Pieces $B$ and $C$ are now reassembled, and players 1,2 , and 3 use the (3,2)-Austin oracle to redivide the result into $B^{\prime}$ and $C^{\prime}$. Since all three think this is a 50-50 division, we still have players 1 and 2 thinking that $A, B^{\prime}, C^{\prime}, D$, and $E$ is an even division, but now player 3 thinks that $B^{\prime}$ and $C^{\prime}$ are tied for second largest (behind $A$ ), or perhaps tied with $A$ for largest.

Player 3 trims $T_{1}$ from $A$ to yield a piece $A^{\prime}$ tied for largest with $B^{\prime}$ and $C^{\prime}$ (and maybe others). Player 4 now trims $T_{2}$ from one of the five pieces $A^{\prime}, B^{\prime}, C^{\prime}, D$, and $E$ to create at least a two-way tie for largest in his estimation. The players choose in the order: $5,4,3,2$, and 1 , subject to the requirement that players 4 and 3 must take the piece they trimmed if it is available. Thus, players 1 and 2 receive untrimmed pieces.

Let $i$ be such that player $i$ received $A^{\prime}$, and let $j$ be such that player $j$ received the piece from which $T_{2}$ was trimmed. Notice that $i, j \neq 2$ (although $i=j$ is possible if player 4 further trimmed piece $A$ to achieve his two-way tie for largest).

This completes the proof of Lemma I.

Lemma II. Assume we have a $(3,2)$-Austin oracle, a piece $T$ of cake, and five players (numbered 1 through 5). Assume that $C-T$ has already been allocated among the five players in an envy-free way, and that player 3 thinks his piece is at least as large as player 4's piece with $T$ added to it, and at least as large as player 
5's piece with $T$ added to it. (That is, player 3 has an irrevocable advantage over players 4 and 5.) Then there exists an allocation of $T$ among the five players so that the allocation of $C$ resulting from this (and the previous allocation of $C-T$ ) is envy-free.

Proof. As in Lemma I, players 1 and 2 begin the procedure by using the generalized version of Austin's scheme to divide $T$ into five pieces that they agree are all the same size. Player 4 now lines up the five pieces - from largest to smallest in his estimation - as $A, B, C, D$, and $E$.

Instead of proceeding as in Lemma I, we now reassemble pieces $A$ and $B$, and have players 1,2 , and 4 use the $(3,2)$-Austin oracle to redivide the result into $A^{\prime}$ and $B^{\prime}$. Since all three think this is a 50-50 division, we still have players 1 and 2 thinking that $A^{\prime}, B^{\prime}, C, D$, and $E$ is an even division, but now player 4 thinks that $A^{\prime}$ and $B^{\prime}$ are tied for largest.

The players now choose in the order: $5,4,3,2$, and 1 . Player 5 envies no one because he is choosing first. Player 4 had a two-way tie for largest, so a largest piece is available for him. Player 3 envies neither 4 nor 5 , because he had an irrevocable advantage over both, and he envies neither 2 nor 1 because he is choosing ahead of them. Players 1 and 2 think all five pieces are the same size.

This completes the proof of Lemma II.

The allocation now proceeds as follows. In Stage 1, Lemma I is applied to the whole cake to obtain an envy-free allocation of part of the cake, with trimmings $T_{1}$ and $T_{2}$ left over (see Figure 1). This also yields $i$ and $j$ (with $i=j$ possible) such that:

1. player 1 thinks his piece is at least as large as player $i$ 's piece with $T_{1}$ added to it, and

2. player 1 thinks his piece is at least as large as player $j$ 's piece with $T_{2}$ added to it.

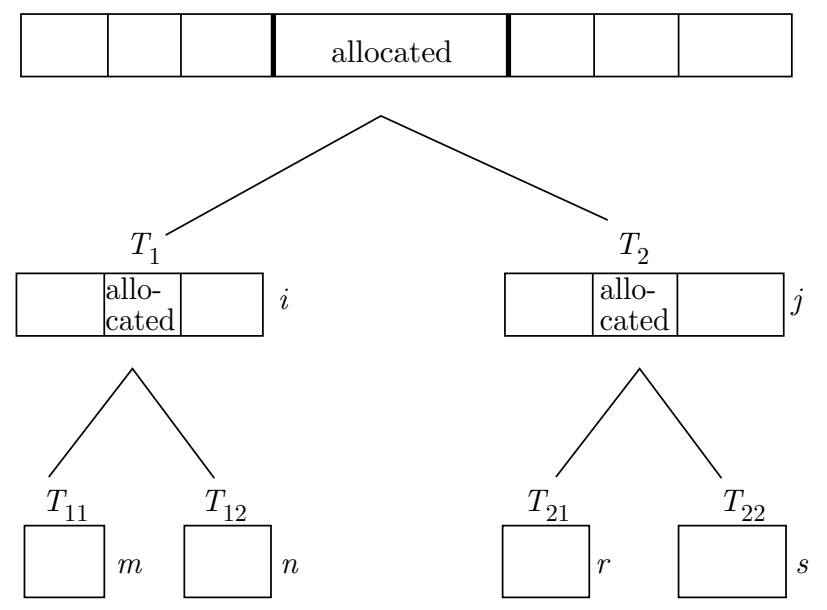

FIGURE 1 
In Stage 2, we allocate part of $T_{1}$ using Lemma I again, but with player $i$ now in the role of player 2 . This yields an envy-free allocation of part of $T_{1}$, with trimmings $T_{11}$ and $T_{12}$ left over. It also yields $m$ and $n$ so that $m$ and $n$ are distinct from $i$, and so that

1. player 1 thinks his piece is at least as large as player $m$ 's piece with $T_{11}$ added to it, and

2. player 1 thinks his piece is at least as large as player $n$ 's piece with $T_{12}$ added to it.

In Stage 3, we allocate all of $T_{11}$ using Lemma II. This is legitimate, because player 1 has an irrevocable advantage over player $i$ and player $m$, and $i \neq m$. Stage 4 allocates $T_{12}$ using Lemma II in an analogous way, and Stages 5, 6, and 7 allocate all of $T_{2}$ in a similar fashion. This completes the proof of Theorem 4.

\section{FormalizATION AND OPEN QUESTIONS}

The history of fair-division algorithms over the last 50 years has proceeded without any precise definition of "discrete algorithm" or of "moving knife scheme". Indeed, positive results (such as "there is a moving-knife scheme for an envy-free allocation among four players") do not appear to require formal rules covering which steps are, and are not, permissible in a moving-knife scheme. Many feel that the informality adds to the subject's simplicity and charm, and we would concur.

But charm and simplicity are not the only factors determining the direction in which mathematics moves or should move. Our analysis in this paper raises several issues that may only admit a resolution via some negative results. While such results may not require complete formalization of what is permissible, they do appear to require partial versions. We will refer to such partial limitations as theses.

For example, suppose one assumes that a permissible step in a discrete scheme consists of a player's cutting a previously generated piece $X$ into two pieces, $Y$ and $Z$, and that there is no limitation on how any other player may value $Y$ and $Z$ - other than that their values sum to the value of $X$. (This is because any choice of such values is consistent with what is known to date about this other player's measure.) Furthermore, suppose that any implementation of a discrete scheme yields a finite sequence of such cuts which partition the original cake into pieces, and that each portion ultimately allocated to a player is a union of such pieces.

It is straightforward to formalize these suppositions in the form of a thesis, which can then be used to show that there is no discrete scheme that will produce an even allocation among any two or more players. That is, for $k \geq 2,(2, k)$-Austin oracles are not superfluous for discrete schemes. In our opinion, most mathematicians would agree that any procedure they would accept as a discrete fair-division algorithm would fit these suppositions, and so they would accept the negative result. However, we don't offer this opinion with a high confidence level-our intention is only to offer an example of an arguable thesis and an associated negative result.

In which direction might limiting theses allow the subject to go next? One possible scenario, which is consistent with what is so far known about discrete algorithms and moving-knife schemes, and which would seem to paint a fairly complete picture, is the following: discrete algorithms do less than moving-knife schemes, but they are equivalent to moving-knife schemes if they are augmented with $(2, k)$-Austin oracles for each $k \geq 2$, and there is no unaugmented moving-knife scheme for di- 
viding a cake into two pieces deemed equal by each of three players. It would seem that new, plausible theses would be required before any part of this picture can be completely filled in.

Additional formalization of some kind also seems to be needed in any attempt to pin down the minimal number of cuts needed for a given type of moving-knife scheme. For example, two cuts are needed in Austin's scheme for dividing a cake in half according to two players (see Section 2). Webb's version [W] of this scheme involves a two-dimensional round cake, in which one player begins with a knife held so as to halve the cake exactly. This player then slowly rotates the knife - while simultaneously shifting it towards, or away from, the center - so as to continue to halve the cake exactly. After a complete 180-degree rotation, the knife must be in its starting position.

As in Austin's scheme, there must be some intermediate point at which the second player also believes the knife halves the cake. At this point, he calls "cut". Webb's version, then, achieves what Austin's did, but with a single cut. In particular, his version can be used to reduce the total number of cuts required in the scheme presented in Section 3.

But this argument seems to fail if what we mean by "cake" is a one-dimensional line segment. One can bend the segment into a circle, pretend it is a pie with no value to the interior, and use Webb's method, but the actual cut then hits the segment in two places, so it seems fair to count it as two cuts. In fact, Webb's method applied to this circle is actually the same as Austin's original scheme applied to the unbent segment. Our feeling at this point is that the number of cuts should be calculated only for one-dimensional cakes, since the degree of connectivity of the underlying space appears to affect the count.

\section{REFERENCES}

[A] A. K. Austin, "Sharing a cake", Mathematical Gazette 6, no. 437 (October 1982), 212-215.

[BT 1] S. J. Brams and A. D. Taylor, "An envy-free cake-division protocol", American Mathematical Monthly 102, no. 1 (January 1995), 9-18. CMP 95:09

[BT 2] S. J. Brams and A. D. Taylor, Fair Division: From Cake-Cutting to Dispute Resolution, Cambridge, UK: Cambridge University Press (1996). CMP 96:10

[BT 3] S. J. Brams and A. D. Taylor, "A note on envy-free cake division", Journal of Combinatorial Theory, Series A 70, no. 1 (April, 1995), 170-173. MR 96b:05014

[BTZ] S. J. Brams, A. D. Taylor, and W. S. Zwicker, "Old and new moving-knife schemes", Mathematical Intelligencer 17, no. 4 (Fall, 1995). CMP 96:05

[N] J. Neyman, "Un theoreme d'existe", C. R. Acad. Sci. Paris 222 (1946), 843-845. MR $7: 457 \mathrm{~h}$

[S] H. Steinhaus, "The problem of fair division", Econometrica 16, no. 1 (January 1948), 101-104.

[St] W. Stromquist, "How to cut a cake fairly", American Mathematical Monthly 87, no. 8 (October 1980), 640-644. Addendum, vol. 88, no. 8 (October 1981), 613-614. MR 81m:05016

[W] W. Webb, "But he got a bigger piece than I did", preprint, n.d.

(S. J. Brams) Department of Politics, New York University, New York, New York 10003

E-mail address: brams@is2.nyu.edu

(A. D. Taylor and W. S. Zwicker) Department of Mathematics, Union College, SchenecTADY, NEW YORK 12308

E-mail address: taylora@gar.union.edu

E-mail address: zwickerw@gar.union.edu 